\title{
MicroRNA-326 functions as a tumor suppressor in colorectal cancer by targeting the nin one binding protein
}

\author{
LEI WU ${ }^{1,2^{*}}$, HUI HUI ${ }^{3 *}$, LI-JUAN WANG ${ }^{4}$, HAO WANG $^{1,2}$, QIU-FANG LIU ${ }^{2}$ and SU-XIA HAN ${ }^{1}$ \\ ${ }^{1}$ Department of Radiotherapy, The First Affiliated Hospital of Medical College of Xi'an Jiaotong University, Xi'an, \\ Shaanxi 710061; Centers of ${ }^{2}$ Radiotherapy and ${ }^{3}$ Gynecological Oncology, Shaanxi Provincial Tumor Hospital, \\ Xi'an, Shaanxi 710068; ${ }^{4}$ Department of Oncology, The First Affiliated Hospital of Medical \\ College of Xi'an Jiaotong University, Xi'an, Shaanxi 710061, P.R. China
}

Received January 2, 2015; Accepted February 27, 2015

DOI: $10.3892 / o r .2015 .3840$

\begin{abstract}
Accumulating evidence has demonstrated that microRNAs (miRNAs) are involved in multiple processes in cancer development and progression. miR-326 has been identified as a tumor suppressor miRNA in several types of human cancer. However, the specific function of miR-326 and its target the nin one binding protein (NOB1) in colorectal carcinoma (CRC) remains unclear. In the present study, we found that miR-326 inhibited cell proliferation, migration and invasion, and induced cell apoptosis and cell cycle arrest of CRC cells by directly targeting NOB1. Furthermore, the upregulation of miR-326 in CRC cells was revealed to be associated with a feedback loop involving downregulation of the NOB1, which mimics the phenotype induced by miR-326. Importantly, we found that the CRC patients with high expression of miR-326 or low expression of NOB1 tend to obtain a better prognosis. Thus, for the first time, we provide convincing evidence that downregulation of miR-326 inhibited tumor proliferation and tumor metastasis by directly targeting NOB1 in CRC. NOB1 and miR-326 could be potential therapeutic targets for CRC.
\end{abstract}

Correspondence to: Professor Su-Xia Han, Department of Radiotherapy, The First Affiliated Hospital of Medical College of Xi'an Jiaotong University, 277 Yanta West Road, Xi'an, Shaanxi 710061, P.R. China

E-mail: sxhan87@163.com

*Contributed equally

Abbreviations: CRC, colorectal cancer; miRNAs, microRNAs; qRT-PCR, quantitative real-time polymerase chain reaction; NOB1, nin one binding protein; 3'-UTR, 3'-untranslated region; MTT, 3-(4,5-dimethylthiazol-2-yl)-2,5-diphenyltetrazolium bromide; MT, mutant; WT, wild-type; PI, propidium iodide

Key words: miR-326, nin one binding protein, proliferation, prognosis, colorectal cancer

\section{Introduction}

Colorectal carcinoma (CRC) is the third most frequently diagnosed malignancy and the third leading cause of mortality among cancer patients in the USA (1). Despite current therapeutic strategies combing adjuvant chemotherapy, surgery, radiotherapy and sometimes immunotherapy, the prognosis of $\mathrm{CRC}$ remains poor. The main reason for the current situation is that most CRC patients have distant metastasis at diagnosis or develop recurrent metastatic CRC following surgical treatment. Furthermore, lack of an accurate prognosis biomarker makes it difficult to predict the patients survival time after surgery. Although recent developments in molecular biology have provided insight into the molecular mechanisms of CRC, the fundamental molecular mechanisms underlying progression and metastasis in CRC have not been fully elucidated. Thus, identification of CRC metastasis-associated molecules may be beneficial for a further prediction of clinical outcomes and may ultimately be used to identify subsets of patients that may benefit from specific targeted therapies.

MicroRNAs (miRNAs) are small non-coding RNAs ( 22 nucleotides in length), transcribed from non-protein coding genes or introns, which regulate gene expression through repressing translation and cleaving their mRNAs by binding to complementary sites in their 3'-untranslated region (3'-UTR) (2). Currently, several aberrant expressed miRNAs have proven to be associated with carcinogenesis, tumor progression and metastasis in CRC and are taken as prognostic indicators for CRC including miR-150 (3), miR-28-5p/-3p (4), miR-200 (5), miR-494 (6) and many others. miR-326 was found downregulated and functioned as a tumor suppressor in multiple types of solid tumor and hematologic neoplasms, such as glioma (7,8), medulloblastoma (9), cholangiocarcinoma (10) and chronic lymphocytic leukemia (11). Yet, the clarification of the function and clinical significance of miR-326 in CRC are still at an early stage.

The yeast nin one binding protein (NOB1) is an evolutionarily conserved protein (12) and is required for the biogenesis and function of 26s proteasome (13). It has been observed that genetic depletion of NOB1 suppresses the process of $20 \mathrm{~S}$ pre-rRNA to mature $18 \mathrm{~S}$ rRNA, producing markedly high levels of the $20 \mathrm{~S}$ pre-RNA with novel degradation 
intermediates (14). These studies showed that the NOB1 played a crucial role in protease function and RNA metabolism. NOB1 expression was found upregulated and it functioned as an oncogenic molecule in various types of solid tumors, such as breast (15), ovarian (16) and non-small cell lung cancer (17), hepatocellular carcinoma (18) and glioma (7). Importantly, NOB1 was also found upregulated in CRC tissues (19). Yet, up to date, the physiological and pathological function of NOB1 in CRC remains unclear and its relationship with miR-326 in CRC has not been examined.

The aim of the present study was to investigate the relationship between miR-326 and NOB1 in CRC, and, furthermore, to identify the effect of miR-326 and NOB1 on cell proliferation, migration and invasion of the CRC cell lines. We also discuss the clinical significance of miR-326 and NOB1 in CRC. We found that miR-326 inhibited cell proliferation, migration and cell invasion, and induced cell apoptosis and cell cycle arrest of CRC cells by directly targeting NOB1. Importantly, we found that the CRC patients with high expression of miR-326 or low expression of NOB1 tend to obtain better prognosis.

\section{Materials and methods}

Patients and tissue samples. The present study was approved by the Research Ethics Committee of Xi'an Jiaotong University. A written informed consent was obtained from all the participating patients. All the specimens were handled and made anonymous according to the ethical and legal standards. A total of 114 patients were enrolled in the present study. Patients received curative resection for CRC at the First Affiliated Hospital, Xi'an Jiaotong University (Xi'an, China). None of the patients that enrolled in the present study received blood transfusion or chemotherapy prior to surgery. The follow-up information of all the participants was updated every 3 months by telephone. The overall survival was defined as the time elapsed from surgery to death. Information regarding the death of the patients was ascertained by their family.

Quantitative reverse transcriptase PCR ( $q R T-P C R)$ assay. The expression of miR-326 in CRC and the corresponding adjacent tissues were detected by qRT-PCR assay. Briefly, the total RNA was extracted from the tissues using TRIzol reagent (Invitrogen, Carlsbad, CA, USA) according to the manufacturer protocol. Then, miRNA expression levels were quantitated using a TaqMan miRNA Real-Time RT-PCR kit (Applied Biosystems) according to the manufacturer protocol. Data were analyzed with 7500 software v.2.0.1 (Applied Biosystems), with the automatic Ct setting for adapting baseline and threshold for $\mathrm{Ct}$ determination. The universal small nuclear RNA U6 (RNU6B) was used as an endogenous control for miRNAs. Each sample was examined in triplicate and the amounts of PCR products produced were non-neoplastic to RNU6B.

MTT assays. Cell proliferation was analyzed in vitro with the tetrazolium salt 3-(4,5-dimethylthiazol-2-yl)-2,5-diphenyltetrazolium bromide (MTT) reagent. Briefly, 2,000 cells from each group were plated in each well of five 96 -well plates in $200 \mu \mathrm{l}$ of medium. To analyze cell proliferation, $20 \mu \mathrm{l}$ of MTT substrate at a concentration of $2.5 \mathrm{mg} / \mathrm{ml}$ in phosphate-buffered saline (PBS) was added to each well. The plates were then returned to a standard tissue incubator for an additional $4 \mathrm{~h}$. The medium was then removed and the cells were solubilized in $150 \mu \mathrm{l}$ of dimethyl sulfoxide for the colorimetric analysis (wavelength, $490 \mathrm{~nm}$ ). One plate was analyzed immediately after the cells adhered ( $4 \mathrm{~h}$ after plating). Then, one plate per day was examined for the next 4 consecutive days.

Cell apoptosis and cell cycle assays. CRC cells were transfected with NOB1 siRNA, miR-326 and their control for $48 \mathrm{~h}$, and the cells were then suspended in an incubation buffer at a density of $1 \times 10^{6}$ cells $/ \mathrm{ml}$. The cells were incubated with Annexin V-FITC and propidium iodide (PI) (BD Biosciences, San Jose, CA, USA) for $15 \mathrm{~min}$ in the dark at room temperature. Cell apoptosis was then analyzed using FACSCalibur (BD Biosciences, San Diego, CA, USA). For cell cycle distribution, the cells of each group were stained with PI and analyzed by flow cytometry using FACSCalibur. For each group, 10,000 events were acquired. The percentage of the cells in G1, S and G2 phases of the cell cycle was calculated.

Cell migration and invasion assays. Cell migration and invasion capacity were measured using Transwell migration assays (Millipore, Billerica, MA, USA) in vitro. The CRC cells were transfected with miR-326 and miR-control for $48 \mathrm{~h}$, and then the cells were suspended in RPMI-1640 with $10 \mathrm{~g} / \mathrm{l} \mathrm{BSA}$ at a density of $1 \times 10^{6}$ cells $/ \mathrm{ml}$. Then, the cell suspensions $(150 \mu \mathrm{l})$ were seeded in the upper chamber with a porous membrane coated with (for the Transwell invasion assay) or without (for the migration assay) Matrigel (BD Biosciences). To attract the cells, $500 \mu \mathrm{l}$ of RPMI-1640 with $10 \%$ serum was added to the lower chamber. After allowing the cells to migrate for $24 \mathrm{~h}$ or to invade for $48 \mathrm{~h}$, the penetrated cells on the filters were fixed in dried methanol and stained with $4 \mathrm{~g} / \mathrm{l}$ crystal violet. The numbers of the migrated or invasive cells were determined from five random fields using a microscope (Olympus) at a magnification of $\mathrm{x} 10$.

Luciferase reporter assay. CRC cells were seeded in 96-well plates at $60 \%$ confluence. After $24 \mathrm{~h}$, the cells were transfected with $120 \mathrm{ng}$ of WT or MT 3'-UTR of PTEN mRNA expression vector pGL3-target-3'UTR and $30 \mathrm{ng}$ of miR-326 mimics using Lipofectamine 2000. The cells were collected $48 \mathrm{~h}$ after the transfection and the luciferase activity was measured using a Dual-Luciferase Reporter Assay System according to the manufacturer protocol (Promega).

Xenograft model in nude mice. For the tumorigenesis assays, we engineered HCT116 cells to stably express high-miR-326 or low-NOB1. Xenograft tumors were generated by subcutaneous injection of HCT116 cells $\left(2 \times 10^{6}\right)$ into the hind limbs of 4-6 weeks old BALB/c athymic nude mice (nu/nu; Animal Center of Xi'an Jiaotong University, Xi'an, China; $n=5$ for each group). All the mice were housed and maintained under specific pathogen-free conditions and all the experiments were approved by the Animal Care and Use Committee of Xi'an Jiaotong University and were performed in accordance with the institutional guidelines. The tumor size was measured using a slide caliper and the tumor volume was determined by 


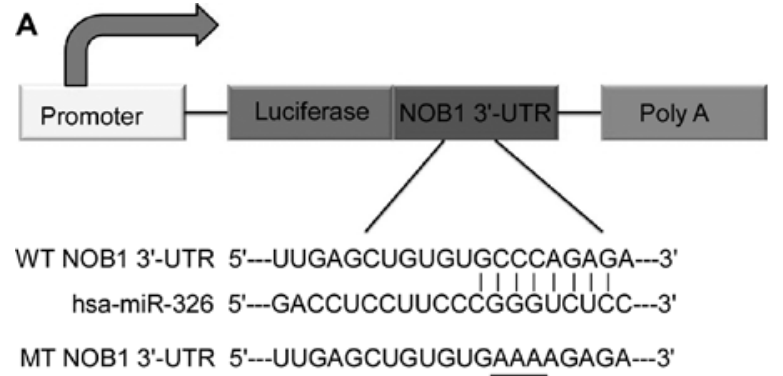

C

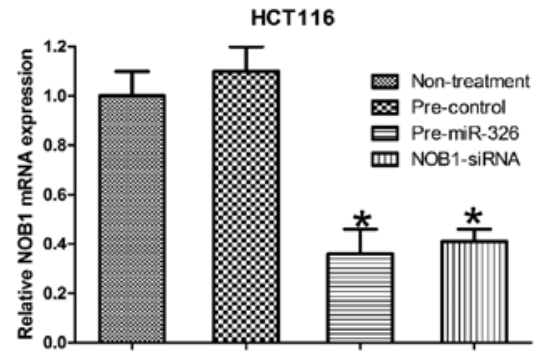

E

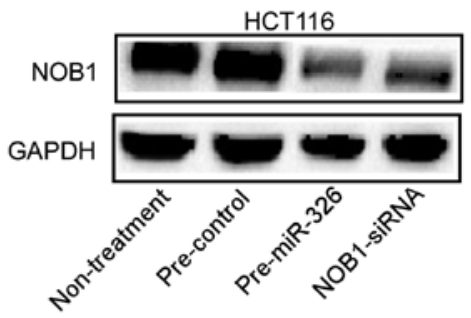

G

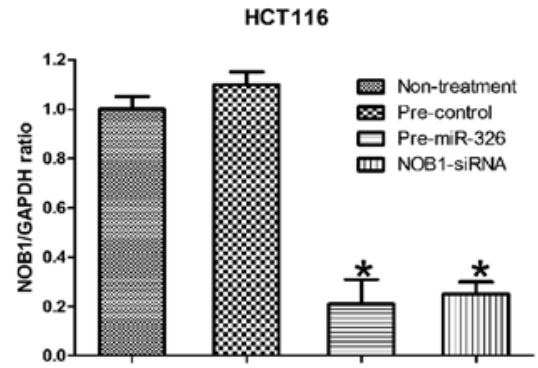

B

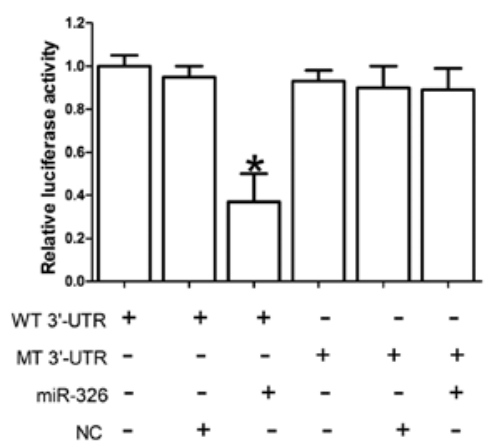

D SW620

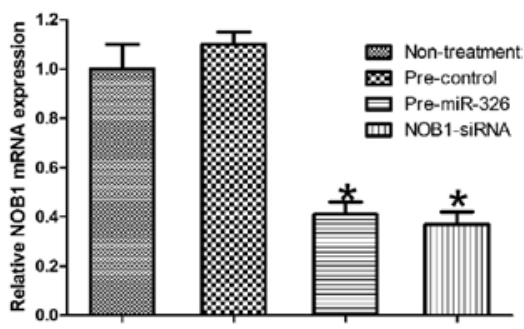

$\mathbf{F}$

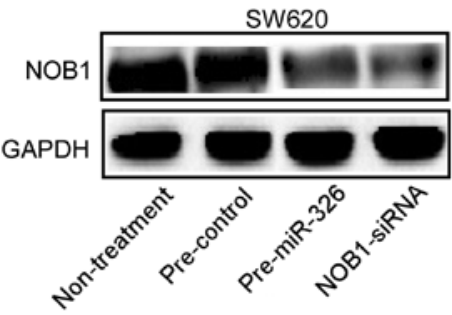

H

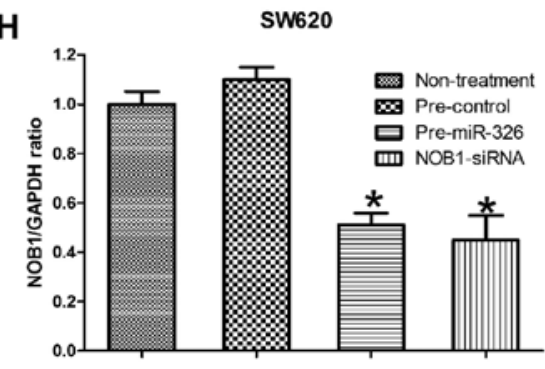

Figure 1. NOB1 is the direct target of miR-326 in CRC cells. (A) The predicted miR-326 binding site in the NOB1 3'-UTR and the diagram of the luciferase reporter plasmids with WT and MT NOB1 3'-UTR. (B) The relative luciferase activity in 293T cells after the plasmid with WT or MT NOB1 3'-UTR co-transfected with miR-326. (C and D) The downregulation of NOB1 mRNA following miR-326 transfection in HCT116 and SW620 was analyzed by qRT-PCR. (E-H) The downregulation of NOB1 protein following miR-326 transfection in HCT116 and SW620 was analyzed by western blotting. Three independent experiments were performed in duplicate. Data are expressed as means \pm SD. A two-tailed Student's t-test was used to analyze the significant differences. "P<0.05. NOB1, nin one binding protein; CRC, colorectal carcinoma; 3'-UTR, 3'-untranslated region; WT, wild-type; MT, mutant.

the formula: $0.44 \times \mathrm{A} \times \mathrm{B} 2$, where A represents the diameter of the base of the tumor and $\mathrm{B}$ represents the corresponding perpendicular value.

Statistical analysis. Statistical analysis was performed using IBM SPSS statistical software (version 21.0). Survival curves were estimated using the Kaplan-Meier method and distributions were evaluated by the long-rank test. Cox proportional hazard models of the factors related to survival were used to calculate RRs and identify the factors that affect survival. The differences in the characteristics between the 2 groups were examined by the $\chi^{2}$ test. All the P-values were determined from the two-sided tests and statistical significance was based on a $\mathrm{P}$-value of $<0.05$.

\section{Results}

NOB1 is the direct target of miR-326 in CRC cells. It has been proven that NOB1 was the direct target of miR-326 in glioma. Considering the developmental stage- and tissue-specific manner of miRNAs, we decided to investigate the relationship between NOB1 and miR-326 in CRC. Using TargetScan, miRanda and PicTar software, NOB1 was identified as a likely target of miR-326, since it contains a putative miR-326 target site in the 3'-UTR. Then, we performed a luciferase reporter assay to further verify whether miR-326 directly targeted the 3'-UTR of NOB1 in CRC. The target sequence of wild-type PTEN 3'-UTR (WT 3'-UTR) or mutant PTEN 3'-UTR (MT 3'-UTR) was cloned into a luciferase reporter 
A

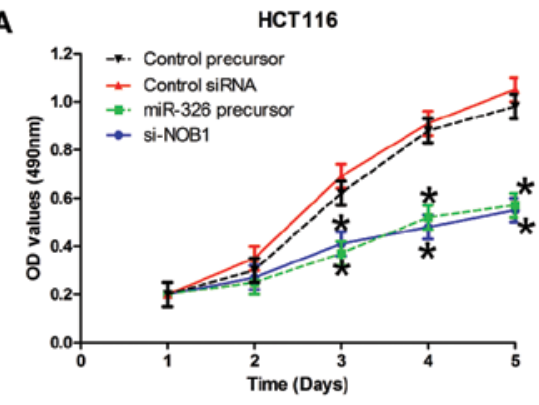

B

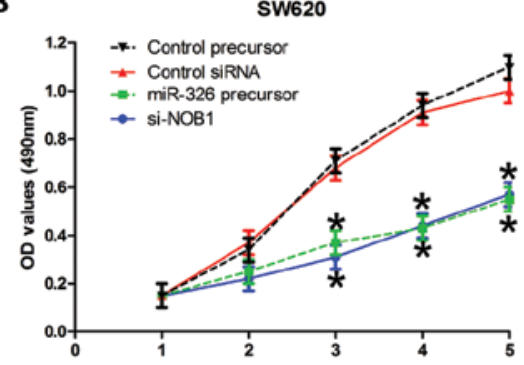

C

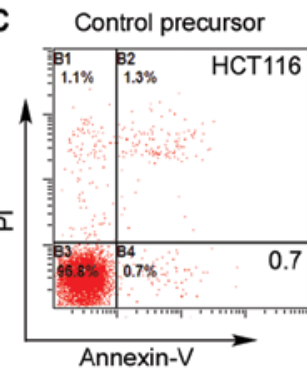

Control siRNA

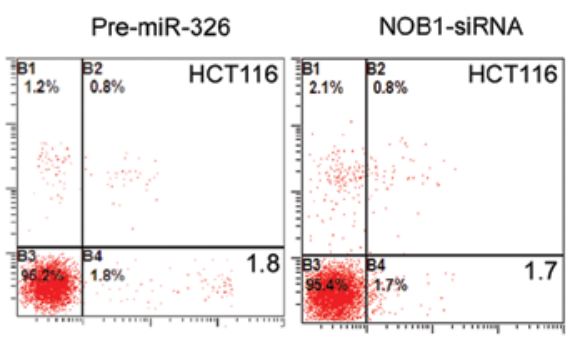

D

D HCT116
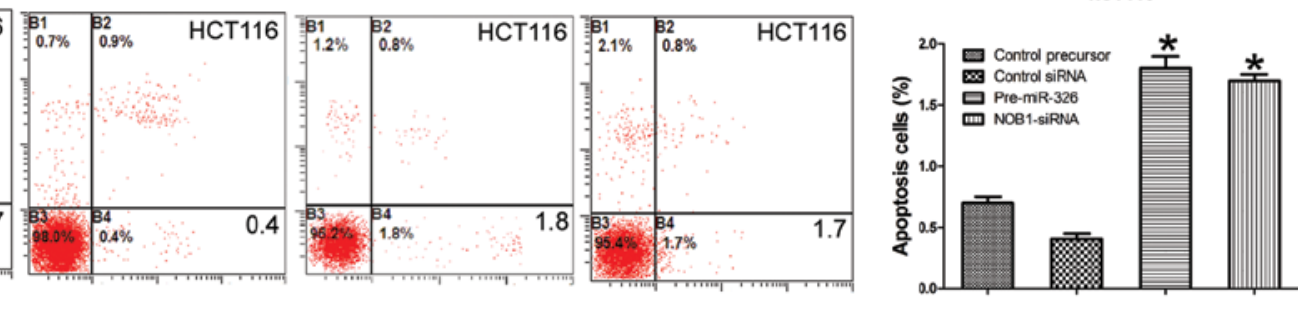

E
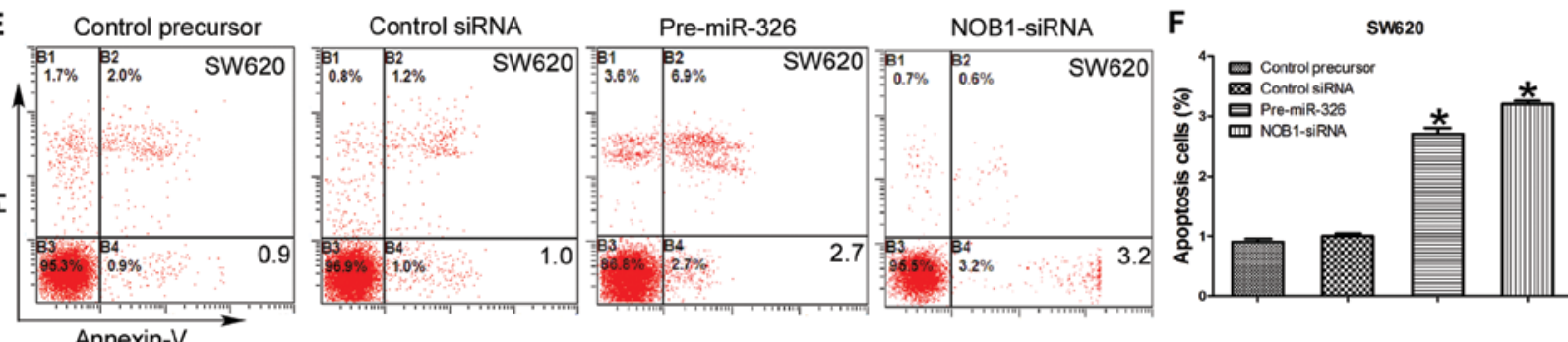

G

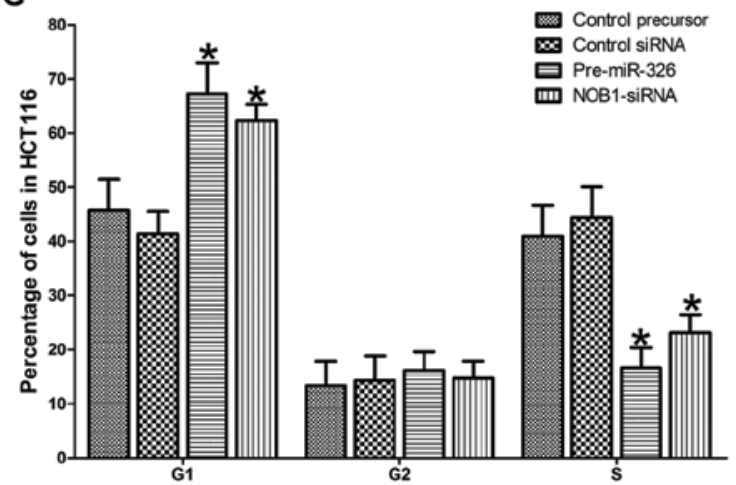

H

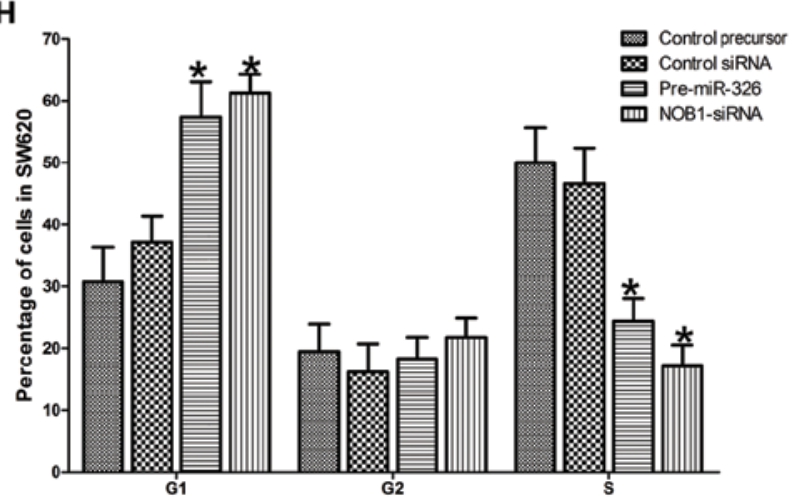

Figure 2. Cell proliferation, cell apoptosis and cell cycle were detected in CRC cells transfected with pre-miR-326 or NOB1 siRNA. (A and B) Effects of miR-326 or NOB1 on cell growth of HCT116 and SW620 cells, which were analyzed by MTT assays. (C-F) Effects of miR-326 or NOB1 on cell apoptosis of HCT116 and SW620 cells. (G and H) Effects of miR-326 or NOB1 on cell cycle of HCT116 and SW620 cells. Three independent experiments were performed in duplicate. Data are expressed as means \pm SD. A two-tailed Student's t-test was used to analyze the significant differences. ${ }^{*}<0.05$. NOB1, nin one binding protein; CRC, colorectal carcinoma.

vector (Fig. 1A). Pre-hsa-miR-326 or non-functional control miR-NC were co-transfected with the reporter vectors into the HEK 293T cells. The miR-326 target sequences and the wildtype NOB1 3'-UTR reduced the relative luciferase activity only when miR-326 was present, but not when the corresponding mutant was introduced with the miR-326 (Fig. 1B). The luciferase reporter data indicated that NOB1 is a specific target of $\operatorname{miR}-326$.

In order to further confirm that NOB1 is the target gene for miR-326 in CRC, qRT-PCR and western blotting were used to detect the expression of NOB1 in CRC cell lines HCT116 and SW620. As expected, the expression of NOB1 at both mRNA and protein level was significantly downregulated in CRC cells transfected with pre-miR-326, the intervention effects of which were similar to NOB1 RNA interference (Fig. 1C-H). Collectively, these results suggest that NOB1 is the direct target gene of miR-326 in CRC cells.

miR-326 inhibits the proliferation of CRC cells by directly targeting NOB1. To analyze the effects of miR-326 on CRC cell proliferation, we transfected miR-326, miR-NC, NOB1- or control-siRNA into HCT116 and SW620 cells. As shown in Fig. 2A and B, ectopic expression of miR-326 inhibited the growth of HCT116 and SW620 cell lines compared to the negative control 5 days after transfection $(\mathrm{P}<0.05)$, and no statistically significant differences in the growth rate between 
A

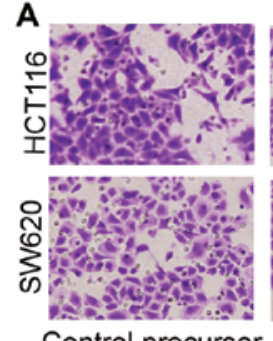$$
\text { ( }
$$

c

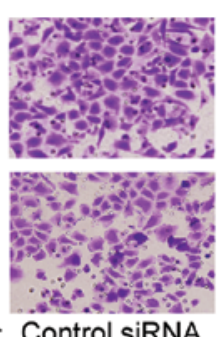

SW620

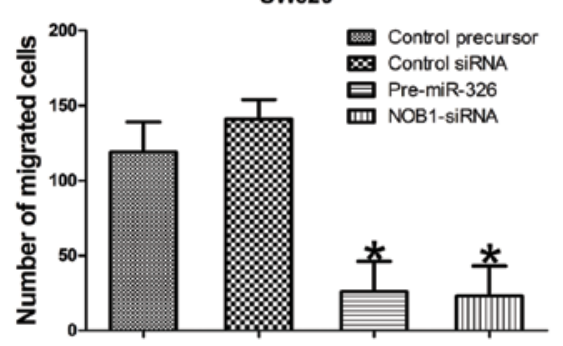

E

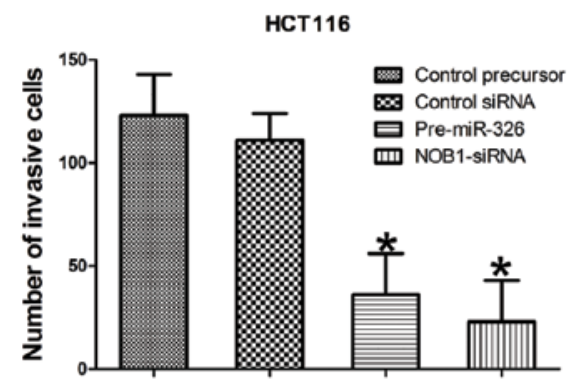

G

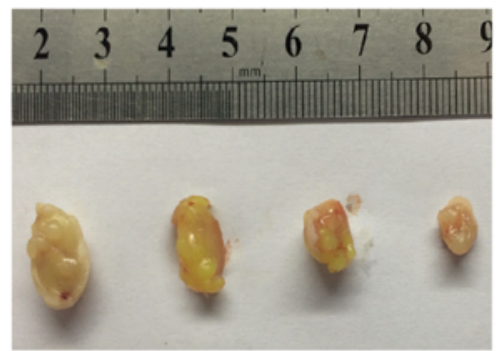

B

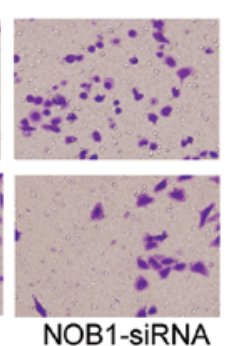

HCT116

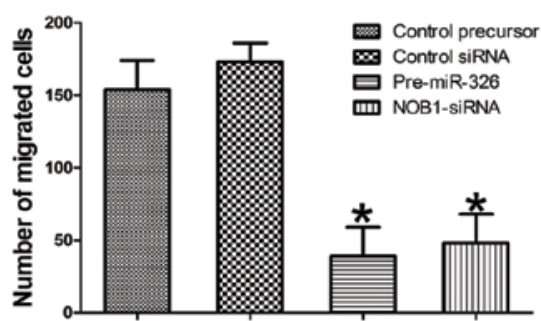

D
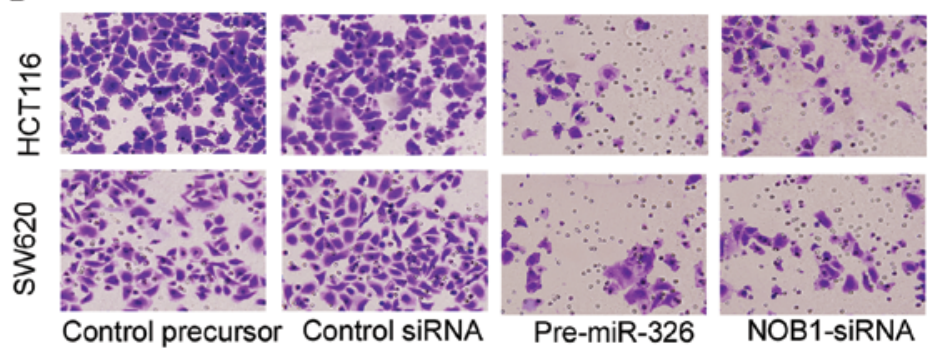

F

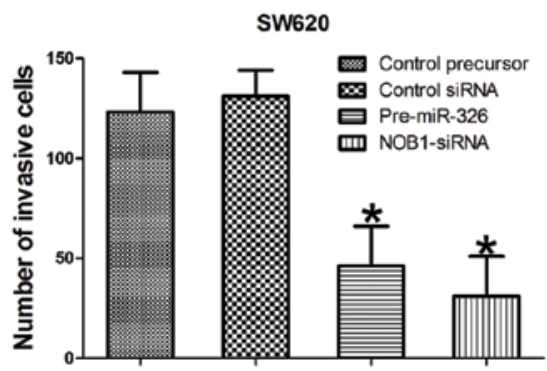

H

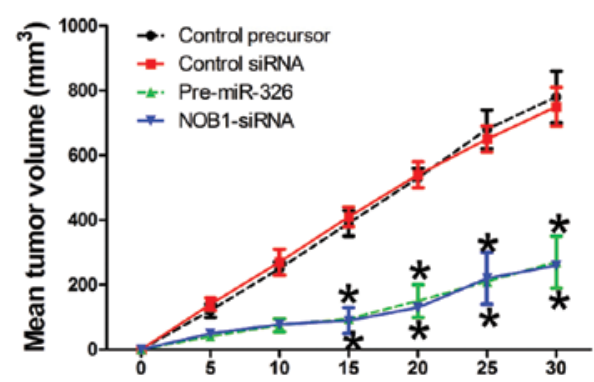

Figure 3. Cell migration, cell invasion and tumor growth were detected in CRC cells transfected with pre-miR-326 or NOB1 siRNA. (A-C) Effects of miR-326 or NOB1 on cell migration of HCT116 and SW620 cells. (D-F) Effects of miR-326 or NOB1 on cell invasion of HCT116 and SW620 cells. (G and H) Effects of miR-326 or NOB1 on tumor growth of nude mice injected with pre-miR-326, miR-NC, NOB1 siRNA and siRNA control (n=3). Tumor volumes were measured on the indicated days. Data points are presented as the mean tumor volume \pm SD. Three independent experiments were performed in duplicate. Data are expressed as means \pm SD. A two-tailed Student's t-test was used to analyze the significant differences. ${ }^{*} \mathrm{P}<0.05$. CRC, colorectal carcinoma; NOB1, nin one binding protein.

the miR-326 overexpressing and the NOB1-siRNA-infected cells was observed. There were no significant differences in the cell growth detected between the miR-NC and the control siRNA. Overall, these data suggest that miR-326 and NOB1 play an important role in regulating the proliferation of CRC cells.

miR-326 induces cell apoptosis and cell cycle arrest in CRC cell lines by directly targeting NOB1. We then examined the effect of miR-326 on apoptosis, HCT116 and SW620 cells were stained with Annexin V and PI after transfection with miR-326, miR-NC, NOB1 siRNA or control siRNA for $72 \mathrm{~h}$. As shown in Fig. 2C-F, the early apoptosis rate in miR-326 and
NOB1 siRNA groups was significantly higher than miR-NC and control siRNA groups $(\mathrm{P}<0.05)$. We found that miR-326 induced early apoptosis in CRC cells by targeting NOB1.

To evaluate the effect of miR-326 on the cell cycle of the CRC cells, we examined the cell cycle distribution by FACS after transfection. Compared with miR- and siRNA-control cells, miR-326 overexpressing and NOB1 silencing cells showed a substantial decrease in the proportion of the cells in the $S$ phase and an increase in the number of the cells in the G1 phase in the HCT116 and SW620 cell lines (Fig. 2G and H). Overall, these results indicate the miR-326 inhibits the proliferation and promotes cell apoptosis of the CRC cells by inducing cell cycle arrest. 
A

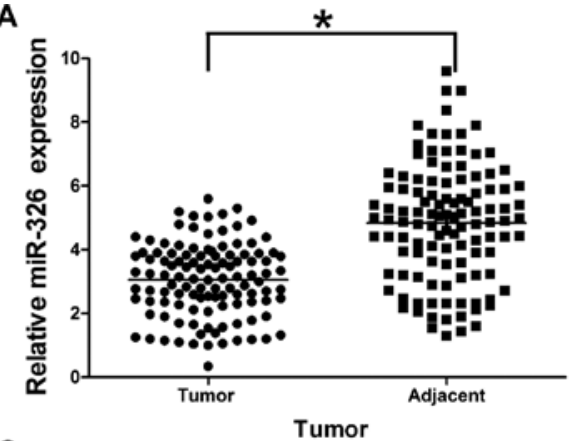

C

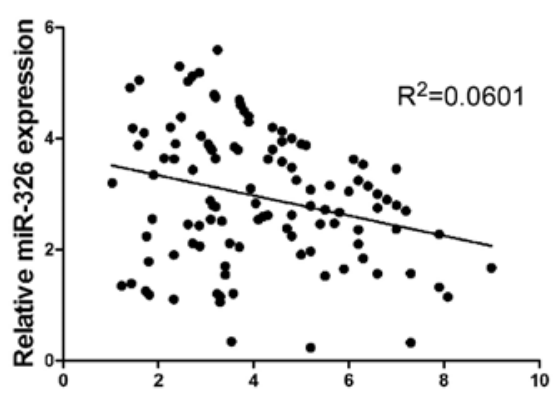

Relative NOB1 mRNA expression

E

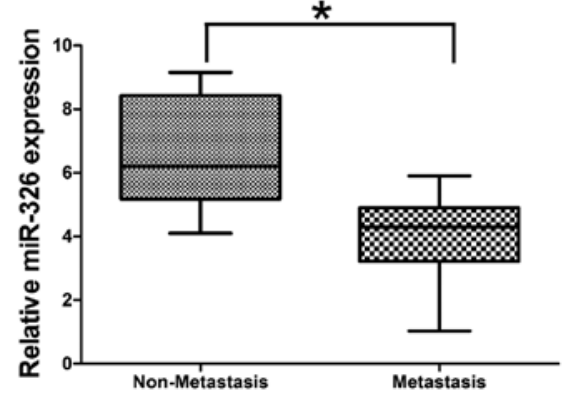

B

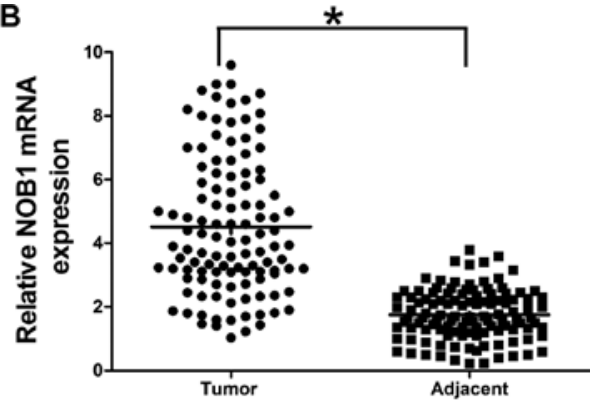

Adjacent tissue

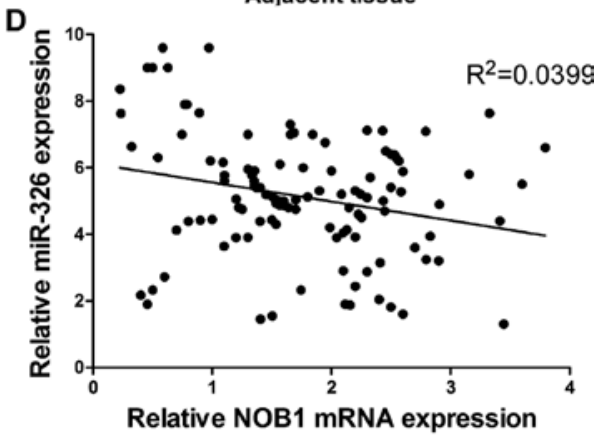

$\mathbf{F}$

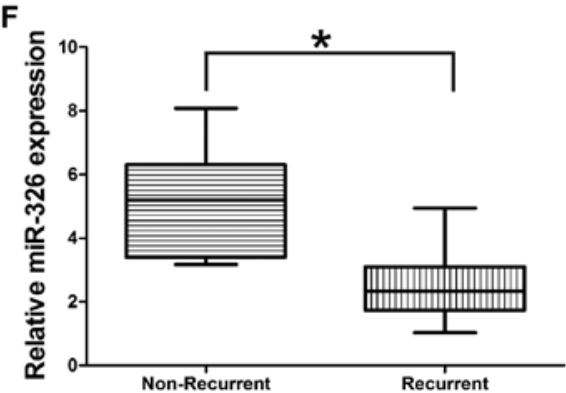

Figure 4. Expression of miR-326 and NOB1 in CRC tissues. (A) Expression of miR-326 in 114 paired CRC tissues and their adjacent normal tissues. (B) Expression of NOB1 mRNA in 114 paired CRC tissues and their adjacent normal tissues. (C) Correlation of miR-494 and PTEN mRNA levels in CRC tissues. (D) Correlation of miR-494 levels and PTEN mRNA levels in CRC adjacent tissues. (E) Expression of miR-326 in tissues of metastatic and non-metastatic CRC patients. (F) Expression of miR-326 in tissues of recurrent and non-recurrent CRC patients. Expression levels of miR-326 and NOB1 were determined by qRT-PCR and normalized against an endogenous control U6 RNA and $\beta$-actin, respectively. "P<0.05. NOB1, nin one binding protein; CRC, colorectal carcinoma.

miR-326 inhibits the migration and invasion of CRC cells by directly targeting NOB1. We then determined whether miR-326 was associated with cell migration and cell invasion of the CRC cells. To test the effect of miR-326 on cell invasion and migration, we used standard Matrigel-coated or uncoated Transwell chamber assays. We found, compared with miR-and siRNA-control cells, that the migration and invasion ability was significantly reduced in HCT116 and SW620 cells that were transfected with miR-326 or NOB1 siRNA (Fig. 3A-F). Collectively, these data suggest that silencing of NOB1 mimics the phenotype induced by overexpression of miR-326 in CRC cells, which further indicates that NOB1 serves as a downstream functional target of miR-326.

miR-326 inhibits tumor formation and tumor growth of CRC cells in vivo. In order to further verify the effect of miR-326 on tumor formation and tumor growth of CRC cells in vivo, we subcutaneously injected the genetically modified (overexpressed miR-326 or NOB1 siRNA) HCT116 cells, as well as their control cells, into the hind limbs of nude mice. Tumor sizes were measured every 5 days; after 30 days, the mice were sacrificed and the tumors were collected. Analysis of the tumor growth curves confirmed that the tumors in the miR-326 or NOB1 siRNA group grew significantly more slowly than those in the miR-NC and siRNA control group (Fig. 3G and H). Collectively, these results further indicate that miR-326 significantly inhibited the tumor formation and tumor growth in human CRC cells, suggesting that miR-326 played a vital function in the tumorigenicity of CRC cells in vitro and in vivo.

miR-326 is downregulated and NOB1 is upregulated in CRC tissues. To further evaluate the clinical significance of miR-326 expression in CRC, we conducted qRT-PCR to measure the miR-326 expression in tumor tissues and their corresponding adjacent tissues of CRC patients. We found that miR-326 expression was significantly downregulated in 114 CRC tumor tissues compared with their corresponding adjacent tissues (Fig. 4A). In contrast, NOB1 expression was significantly upregulated in CRC tumor tissues compared with their corresponding adjacent tissues (Fig. 4B), which was consistent with previous literature. More importantly, statistically significant inverse correlations were revealed by Spearman's 

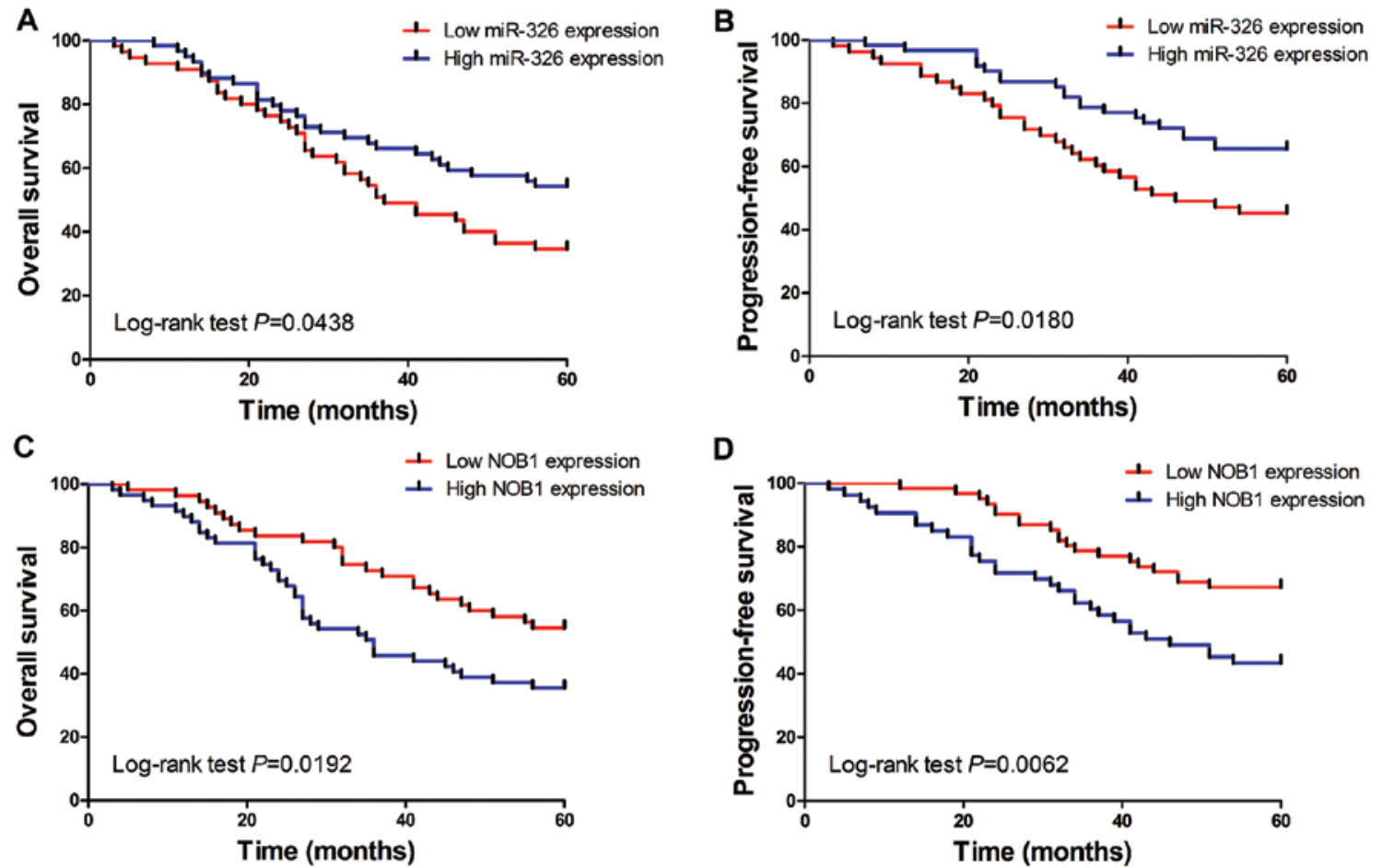

Figure 5. Clinical significance of miR-326 and NOB1 in CRC patients. (A) Overall survival curves for low and high expression of miR-326 in CRC patients. (B) Progression-free survival curves for low and high expression of miR-326 in CRC patients. (C) Overall survival curves for low and high expression of NOB1 in CRC patients. (D) Progression-free survival curves for low and high expression of NOB1 in CRC patients. Expression levels of miR-326 and NOB1 was determined by qRT-PCR and normalized against an endogenous control U6 RNA. "P<0.05. NOB1, nin one binding protein; CRC, colorectal carcinoma.

Table I. Cox regression analysis of prognostic factors for overall survival in CRC patients $(\mathrm{n}=114)$.

\begin{tabular}{|c|c|c|c|c|c|c|}
\hline & \multicolumn{3}{|c|}{ Univariate } & \multicolumn{3}{|c|}{ Multivariate } \\
\hline & HR & $95 \% \mathrm{CI}$ & P-value & HR & $95 \% \mathrm{CI}$ & P-value \\
\hline miR-326 expression & 0.679 & $0.329-0.875$ & $0.018^{\mathrm{a}}$ & 0.582 & $0.282-0.798$ & $0.011^{\mathrm{a}}$ \\
\hline NOB1 expression & 3.271 & $1.478-4.182$ & $0.031^{\mathrm{a}}$ & 2.894 & $1.391-3.829$ & $0.037^{\mathrm{a}}$ \\
\hline Age (years) & 1.723 & $0.674-2.147$ & 0.421 & 2.007 & $0.771-2.317$ & 0.328 \\
\hline Gender & 1.682 & $0.819-2.781$ & 0.391 & 1.713 & $0.671-2.178$ & 0.482 \\
\hline Tumor location & 2.398 & $0.519-3.298$ & 0.127 & 2.128 & $0.661-2.891$ & 0.228 \\
\hline TNM stage & 3.751 & $1.874-5.118$ & $0.008^{\mathrm{a}}$ & 4.217 & $2.114-5.981$ & $0.011^{\mathrm{a}}$ \\
\hline Differentiation & 2.511 & $0.754-3.187$ & 0.073 & 1.927 & $0.678-3.722$ & 0.067 \\
\hline
\end{tabular}

95\% CI indicates $95 \%$ confidence interval. ${ }^{\mathrm{a}} \mathrm{P} \leq 0.05$. $\mathrm{CRC}$, colorectal carcinoma; HR, hazard ratio; NOB1, nin one binding protein; TNM, tumor-node-metastasis.

correlation analysis between mRNA levels of miR-326 and NOB1 in CRC tumor and adjacent specimens (Fig. 4C and D; $\mathrm{r}=-0.2452 ; \mathrm{P}=0.0086$ and $\mathrm{r}=-0.1998 ; \mathrm{P}=0.0331)$. Collectively, these results suggest that miR-326 plays a tumor suppressor role and NOB1 has an oncogenic role in CRC.

miR-326 is associated with tumor metastasis and recurrence in $C R C$ patients. We then determined whether miR-326 was associated with tumor metastasis and recurrence. Tumor specimens were divided into two groups according to their metastatic or recurrent status. Our data showed that miR-326 expression decreased in the metastatic group (Fig. 4E).
Moreover, miR-326 levels were also significantly decreased in the specimens obtained from the patients who suffered CRC recurrence (Fig. 4F). Collectively, our findings showed that downregulation of miR-326 may increase the risk of metastasis and recurrence in CRC patients.

miR-326 is associated with survival and prognosis in CRC patients. In order to further determine the clinical significance of miR-326 in CRC, we split the 114 patients into two groups based on miR-326 or NOB1 expression levels (low vs. high) with their median expression levels as a cut-off point. The Kaplan-Meier analysis revealed that low miR-326 expression 
Table II. Cox regression analysis of prognostic factors progression-free survival in CRC patients $(n=114)$.

\begin{tabular}{|c|c|c|c|c|c|c|}
\hline & \multicolumn{3}{|c|}{ Univariate } & \multicolumn{3}{|c|}{ Multivariate } \\
\hline & HR & $95 \% \mathrm{CI}$ & P-value & HR & $95 \% \mathrm{CI}$ & P-value \\
\hline miR-326 expression & 0.718 & $0.327-0.911$ & $0.021^{\mathrm{a}}$ & 0.811 & $0.418-0.951$ & $0.017^{\mathrm{a}}$ \\
\hline NOB1 expression & 3.518 & $1.587-4.291$ & $0.027^{\mathrm{a}}$ & 3.891 & $1.981-5.155$ & $0.032^{\mathrm{a}}$ \\
\hline Age (years) & 1.784 & $0.781-2.871$ & 0.325 & 2.214 & $0.881-2.959$ & 0.411 \\
\hline Gender & 2.187 & $0.891-3.128$ & 0.237 & 1.895 & $0.711-2.623$ & 0.229 \\
\hline Tumor location & 1.671 & $0.687-2.887$ & 0.229 & 2.181 & $0.667-3.193$ & 0.14 \\
\hline TNM stage & 4.789 & $1.871-5.918$ & $0.014^{\mathrm{a}}$ & 3.726 & $1.771-4.918$ & $0.007^{\mathrm{a}}$ \\
\hline Differentiation & 2.198 & $0.518-3.115$ & 0.074 & 2.481 & $0.784-3.214$ & 0.051 \\
\hline
\end{tabular}

95\% CI indicates 95\% confidence interval. ${ }^{\mathrm{a}} \mathrm{P} \leq 0.05$. CRC, colorectal carcinoma; HR, hazard ratio; NOB1, nin one binding protein; TNM, tumor-node-metastasis.

was significantly correlated with reduced overall and progression-free survival in $114 \mathrm{CRC}$ patients (Fig. 5A and B; log-rank test, $\mathrm{P}=0.0438$ and $\mathrm{P}=0.0180$, respectively). Whereas, the patients with low NOB1 expression tended to obtain better overall and progression-free survival time, than patients with high NOB1 expression (Fig. 5C and D; log-rank test, $\mathrm{P}=0.0192$ and $\mathrm{P}=0.0062$, respectively).

Next, the univariate analysis demonstrated that the overall and progression-free survival of CRC patients was associated with tumor-node-metastasis (TNM) stage, NOB1 and miR-326 expression (Tables I and II). To test whether the prognostic value of miR-326 and NOB1 was independent of other clinicopathological parameters for poor overall and progression-free survival in CRC patients, a multivariate analysis was performed using a Cox proportional hazard model. Multivariate analysis including miR-326 expression, NOB1 expression, age, gender, tumor location, TNM stage and differentiation status, demonstrated that miR-494 or NOB1 expression was an independent prognostic biomarker for poor overall and progression-free survival in CRC patients (Tables I and II). These results suggest that patients with high-miR-326 expression tend to obtain a better prognosis than patients with low-miR-326 expression, yet the patients with high NOB1 expression tend to obtain a worse prognosis than the patients with low-NOB1 expression. Statistically significant results were also obtained for the TNM stage and lymph node metastasis, where all the other parameters were not independent prognostic biomarkers for overall and progression-free survival in CRC patients. Collectively, these results indicate that miR-326 or its target NOB1 is an independent biomarker for survival and prognosis of CRC patients.

\section{Discussion}

MicroRNAs (miRNAs) are unique in their ability to regulate many protein-coding genes. Several miRNA have been identified as candidate components of oncogene and tumor suppressor networks in CRC, these miRNA and their targets play an important role in the occurrence and development of CRC. The changes in miRNA profiling are associated with almost all the aspects of CRC cell biology, such as cell proliferation (20), angiogenesis (21), apoptosis (22), cell cycle (23), migration and invasion (6). In the present study, we focused on the role of miR-326 in CRC, which has been proven to be a tumor suppressor miRNA in multiple types of cancer. We found that miR-326 inhibited cell proliferation, migration and invasion of CRC cells, yet it induced cell apoptosis and cell cycle arrested of CRC cells. These results indicate that miR-326 also functions as a tumor suppressor in CRC. Furthermore, the upregulation of miR-326 in the CRC cells was revealed to be associated with a feedback loop involving downregulation NOB1 which mimics the phenotype induced by miR-326. We identified miR-326 downregulation or NOB1 upregulation, which was usually associated with poor survival and prognosis of CRC patients.

A previous study confirmed that NOB1, an essential protein, was associated with $26 \mathrm{~S}$ proteasome and hence played an essential role in the universal biological process. In the present study, we showed that miR-326 inhibited carcinogenesis and progression by blocking a novel target, NOB1, both in vitro and in vivo. When the cell cycle of CRC cells was assessed by FACS, we observed that upregulated miR-326 expression or downregulated NOB1 expression induced a significant decrease in the $\mathrm{S}$ phase and an increase in the G1 phase population in the CRC cells with significant cell proliferation arrest. The growth inhibitory effect was further confirmed by MTT assays and nude mouse xenograft assays, indicating that miR-326 and NOB1 are crucial for proliferation and carcinogenesis of human CRC. In addition, upregulated miR-326 expression or downregulated NOB1 expression inhibited cell migration and invasion of CRC cells. Metastasis is the major reason for poor prognosis of $\mathrm{CRC}$ patients. Thus, our findings indicated that miR-326 and/or NOB1 may be a potential therapeutic target for CRC. Thus, we investigated the clinical significance of miR-326 and NOB1. As a previous study confirmed NOB1 expression was upregulated in tissues of CRC and promoted tumor growth and carcinogenesis of CRC $(24,25)$, thus we focused on whether miR-326 and/or NOB1 could be the prognostic indicators for CRC. We found that miR-326 downregulation or NOB1 upregulation was usually associated with poor 
survival. Univariate and multivariate results showed that a high miR-326 expression or a low NOB1 expression was associated with poor prognosis of CRC patients. Each was an independent prognostic biomarker for CRC.

Since the identification of NOB1 protein, increased NOB1 expression has been reported in ovarian (16), colorectal (24), breast (15), thyroid (26) and hepatocellular carcinoma (18). As the genetic depletion of NOB1 strongly suppressed the processing of $20 \mathrm{~S}$ pre-rRNA to mature $18 \mathrm{~S} \mathrm{rRNA}$, producing markedly high levels of $20 \mathrm{~S}$ pre-RNA with novel degradation intermediates (14) the effect corrects the RNA synthesis and affects the cell cycle regulation (27), since it is necessary for synthesis of rRNA for cell cycle process $(28,29)$. Moreover, NOB1 also plays an important role in proteasomes by forming a complex between $19 \mathrm{~S}$ regulatory particle of $26 \mathrm{~S}$ proteasome, where the latter catalyzes the protein degradation through the ubiquitin proteasome pathway for cell cycle progression $(30,31)$. All these studies indicate that NOB1 expression has an essential role in cell cycle regulation. Our study supported the pivotal role of NOB1 in cell cycle regulation of CRC cells. Our results also indicated that NOB1 affected cell proliferation and tumor growth of CRC in vivo and in vitro by regulating the cell cycle.

Recently, several profiling analyses reported that NOB1 was involved in carcinogenesis and tumor metastasis. Notably, NOB1 was found to play a critical role in tumor metastasis of clear cell renal cell carcinoma (32). Consistent with a previous study, we also found that NOB1 was associated with cell migration and invasion of the CRC cell. Yet, the molecular mechanisms underlying this phenomenon are still largely unknown and need further specific investigation. Several miRNAs have also been proven to be associated with carcinogenesis, progression and metastasis of CRC. Recently, miR-494 was found to be associated with tumor metastasis and tumor aggressiveness of CRC (6). Moreover, miR-139-5p suppressed CRC cell invasion and metastasis by targeting AMFR and NOTCH1 (33). Our study also indicates that miR-326 plays an important role in cell metastasis by directly targeting NOB1. We identified that miR-326 and NOB1 were the metastasis-associated molecules and may be considered as potential therapeutic targets for CRC. These results shed new light on the understanding of the molecular mechanism underlying the metastasis of CRC.

Though miR-326 was found downregulated and as it functions as a tumor suppressor in multiple types of solid tumor and hematologic neoplasms, the biological effects and clinical significance of miR-326 in CRC are still not fully understood. Consistent with the above studies, our results identified that miR-326 functioned as a tumor suppressor in CRC. miR-326 inhibited cell proliferation, cell migration and cell invasion of CRC cells. Importantly, miR-326 was also an independent prognostic biomarker for CRC patients. However, a recent report exists with contradictory conclusion (34). In the present study, plasma miR-326 was found upregulated in CRC, and high expression of plasma miR-326 was associated with a decreased overall and progression-free survival, indicating miR-326 may play an oncogenic role in CRC. The discrepancies between the studies may reflect the different cohorts selected for each study. We focused on the role that miR-326 played in CRC cells and the tissues of the CRC patients. Yet other authors put their emphasis on whether plasma miRNAs before treatment may serve as non-invasive markers predicting the outcome in the metastatic CRC patients treated with 5-FU and oxaliplatin-based chemotherapy. Circulating expression of miRNAs has been described in many solid cancers including CRC, but it is still difficult to differentiate whether circulating miRNAs expression is specifically associated with CRC itself or if this is a common phenomenon that manifests during progression of any cancer as a result of perturbations in host immune response (35). Thus, when investigating the role that circulating miRNA played in CRC, we must make clear whether a direct correlation exists between tissue and serum levels of miRNA and their specificity in CRC. Considering the contradicting conclusion, retrospective or prospective studies with a sufficient number of samples should be conducted to make clear the role that miR-326 plays in CRC.

In summary, we showed that miR-326 was downregulated and its target NOB1 was upregulated in CRC tissues. miR-326 decreased carcinogenesis and metastasis of CRC cells through directly targeting NOB1. Clinically, miR-326 or NOB1 was independently prognostic biomarker for CRC patients. Our findings suggest that exogenous overexpression of miR-326 may be considered as a promising strategy for targeted therapies in CRC.

\section{Acknowledgements}

The present study was supported in part by the Health Research Projects 2014B1 of Shaanxi Province Health and the Family Planning Commission. The authors would like to thank the local doctors and the patients who participated in the present study.

\section{References}

1. Siegel R, Ma J, Zou Z and Jemal A: Cancer statistics, 2014. CA Cancer J Clin 64: 9-29, 2014.

2. Kumar MS, Lu J, Mercer KL, Golub TR and Jacks T: Impaired microRNA processing enhances cellular transformation and tumorigenesis. Nat Genet 39: 673-677, 2007.

3. Ma Y, Zhang P, Wang F, Zhang H, Yang J, Peng J, Liu W and Qin H: miR-150 as a potential biomarker associated with prognosis and therapeutic outcome in colorectal cancer. Gut 61: 1447-1453, 2012.

4. Almeida MI, Nicoloso MS, Zeng L, et al: Strand-specific miR-28-5p and miR-28-3p have distinct effects in colorectal cancer cells. Gastroenterology 142: 886-896.e9, 2012.

5. Pichler M, Ress AL, Winter E, Stiegelbauer V, Karbiener M, Schwarzenbacher D, Scheideler M, Ivan C, Jahn SW, Kiesslich T, et al: MiR-200a regulates epithelial to mesenchymal transition-related gene expression and determines prognosis in colorectal cancer patients. Br J Cancer 110: 1614-1621, 2014

6. Sun HB, Chen X, Ji H, Wu T, Lu HW, Zhang Y, Li H and $\mathrm{Li} Y \mathrm{YM}$ : miR-494 is an independent prognostic factor and promotes cell migration and invasion in colorectal cancer by directly targeting PTEN. Int J Oncol 45: 2486-2494, 2014.

7. Zhou J, Xu T, Yan Y, Qin R, Wang H, Zhang X, Huang Y, Wang Y, Lu Y, Fu D, et al: MicroRNA-326 functions as a tumor suppressor in glioma by targeting the Nin one binding protein (NOB1). PLoS One 8: e68469, 2013.

8. Du W, Liu X, Chen L, et al: Targeting the SMO oncogene by miR-326 inhibits glioma biological behaviors and stemness. Neuro Oncol 17: 243-253, 2015. 
9. Ferretti E, De Smaele E, Miele E, Laneve P, Po A, Pelloni M, Paganelli A, Di Marcotullio L, Caffarelli E, Screpanti I, et al: Concerted microRNA control of Hedgehog signalling in cerebellar neuronal progenitor and tumour cells. EMBO J 27: 2616-2627, 2008.

10. Meng F, Henson R, Lang M, Wehbe H, Maheshwari S, Mendell JT, Jiang J, Schmittgen TD and Patel T: Involvement of human micro-RNA in growth and response to chemotherapy in human cholangiocarcinoma cell lines. Gastroenterology 130 : 2113-2129, 2006

11. Babashah S, Sadeghizadeh M, Hajifathali A, Tavirani MR, Zomorod MS, Ghadiani M and Soleimani M: Targeting of the signal transducer Smo links microRNA-326 to the oncogenic Hedgehog pathway in CD34 ${ }^{+} \mathrm{CML}$ stem/progenitor cells. Int J Cancer 133: 579-589, 2013.

12. Makarova KS, Aravind L, Galperin MY, Grishin NV, Tatusov RL, Wolf YI and Koonin EV: Comparative genomics of the Archaea (Euryarchaeota): Evolution of conserved protein families, the stable core, and the variable shell. Genome Res 9: 608-628, 1999.

13. Zhang Y, Ni J, Zhou G, Yuan J, Ren W, Shan Y, Tang W, Yu L and Zhao S: Cloning, expression and characterization of the human NOB1 gene. Mol Biol Rep 32: 185-189, 2005.

14. Fatica A, Oeffinger M, Dlakić M and Tollervey D: Nob1p is required for cleavage of the $3^{\prime}$ end of $18 \mathrm{~S}$ rRNA. Mol Cell Biol 23: 1798-1807, 2003.

15. Huang WY, Chen DH, Ning L and Wang LW: siRNA mediated silencing of NIN1/RPN12 binding protein 1 homolog inhibits proliferation and growth of breast cancer cells. Asian Pac J Cancer Prev 13: 1823-1827, 2012.

16. Lin Y, Peng S, Yu H, Teng H and Cui M: RNAi-mediated downregulation of $N O B 1$ suppresses the growth and colony-formation ability of human ovarian cancer cells. Med Oncol 29: 311-317, 2012.

17. Li Y, Ma C, Qian M, Wen Z, Jing H and Qian D: Downregulation of NOB1 suppresses the proliferation and tumor growth of non-small cell lung cancer in vitro and in vivo. Oncol Rep 31: 1271-1276, 2014.

18. Lu Z, Guo Q, Shi A, Xie F and Lu Q: Downregulation of NIN/RPN12 binding protein inhibit the growth of human hepatocellular carcinoma cells. Mol Biol Rep 39: 501-507, 2012.

19. He XW, Tao F, Luo SS and Yu XK: Effect of lentivirus-mediated NOB1 gene silencing by RNA interference on proliferation and apoptosis of human colon cancer cells. Zhonghua Wei Chang Wai Ke Za Zhi 15: 1182-1186, 2012 (In Chinese).

20. Gao F and Wang W: MicroRNA-96 promotes the proliferation of colorectal cancer cells and targets tumor protein p53 inducible nuclear protein S1, forkhead box protein O1 (FOXO1) and FOXO3a. Mol Med Rep 11: 1200-1206, 2015.

21. Xue G, Yan HL, Zhang Y, Hao LQ, Zhu XT, Mei Q and Sun SH: c-Myc-mediated repression of miR-15-16 in hypoxia is induced by increased HIF-2 $\alpha$ and promotes tumor angiogenesis and metastasis by upregulating FGF2. Oncogene: Apr 7, 2014 (Epub ahead of print). doi: 10.1038/onc.2014.82.
22. Fernandez S, Risolino M, Mandia N, et al: miR-340 inhibits tumor cell proliferation and induces apoptosis by targeting multiple negative regulators of p27 in non-small cell lung cancer. Oncogene 0: 2014

23. Li T, Yang J, Lv X, Liu K, Gao C, Xing Y and Xi T: miR-155 regulates the proliferation and cell cycle of colorectal carcinoma cells by targeting E2F2. Biotechnol Lett 36: 1743-1752, 2014.

24. Wu DP and He XW: Expression of NOB1 and its significance in colorectal cancer. Nan Fang Yi Ke Da Xue Xue Bao 32: 420-422, 2012 (In Chinese).

25. Liu Y, Huang H, Yuan B, Zhuang LY, Luo TP and Zhang Q: Lentivirus-mediated knockdown of NOB1 suppresses the proliferation of colon cancer cells. Z Gastroenterol 52: 429-435, 2014

26. Meng W, Wang PS, Liu J, Xue S, Wang GM, Meng XY and Chen G: Adenovirus-mediated siRNA targeting NOB1 inhibits tumor growth and enhances radiosensitivity of human papillary thyroid carcinoma in vitro and in vivo. Oncol Rep 32: 2411-2420, 2014.

27. Granneman S, Nandineni MR and Baserga SJ: The putative NTPase Fap7 mediates cytoplasmic 20S pre-rRNA processing through a direct interaction with Rps14. Mol Cell Biol 25: 10352-10364, 2005.

28. Raychaudhuri S, Fontanes V, Barat B and Dasgupta A: Activation of ribosomal RNA transcription by hepatitis $C$ virus involves upstream binding factor phosphorylation via induction of cyclin D1. Cancer Res 69: 2057-2064, 2009.

29. Meraner J, Lechner M, Loidl A, Goralik-Schramel M, Voit R, Grummt I and Loidl P: Acetylation of UBF changes during the cell cycle and regulates the interaction of UBF with RNA polymerase I. Nucleic Acids Res 34: 1798-1806, 2006.

30. Fasanaro P, Capogrossi MC and Martelli F: Regulation of the endothelial cell cycle by the ubiquitin-proteasome system. Cardiovasc Res 85: 272-280, 2010.

31. Xu G, Bernaudo S, Fu G, Lee DY, Yang BB and Peng C: Cyclin G2 is degraded through the ubiquitin-proteasome pathway and mediates the antiproliferative effect of activin receptor-like kinase 7. Mol Biol Cell 19: 4968-4979, 2008.

32. Li W, Liu M, Feng Y, Xu YF, Huang YF, Che JP, Wang GC, Yao XD and Zheng JH: Downregulated miR-646 in clear cell renal carcinoma correlated with tumour metastasis by targeting the nin one binding protein (NOB1). Br J Cancer 111: 1188-1200, 2014.

33. Song M, Yin Y, Zhang J, Zhang B, Bian Z, Quan C, Zhou L, Hu Y, Wang Q, Ni S, et al: MiR-139-5p inhibits migration and invasion of colorectal cancer by downregulating AMFR and NOTCH1. Protein Cell 5: 851-861, 2014.

34. Kjersem JB, Ikdahl T, Lingjaerde OC, Guren T, Tveit KM and Kure EH: Plasma microRNAs predicting clinical outcome in metastatic colorectal cancer patients receiving first-line oxaliplatin-based treatment. Mol Oncol 8: 59-67, 2014.

35. No authors listed: MicroRNA miR-133 represses HERG $\mathrm{K}^{+}$ channel expression contributing to QT prolongation in diabetic hearts. J Biol Chem 286: 28656, 2011. 